\title{
The Internet of Things Technology Application Analysis in Single-Chip Computer Teaching Reform
}

\author{
Wai Cui \\ The Engineering \& Technical College of Chengdu University of Technology, China
}

Keywords: Single chip computer teaching, The teaching reform, The Internet of things technology, Application analysis.

\begin{abstract}
Single chip computer teaching is strong comprehensive, practical, strong physical characteristics. Traditional SCM teaching can't adapt to the training objectives of education under the new situation, this paper discusses on the necessity of the single chip computer teaching reform, teaching contents and teaching reform IoT technology combination, strengthen the reform of experiment practice link and examination form has made some exploration, etc.
\end{abstract}

\section{Introduction}

Along with the development of integrated circuit technology and the Internet of things technology and system on chip (SOC), the rapid development of modern technology, the single chip microcomputer has been gradually from four, eight transition to a 32bit, ARM as the core of single chip microcomputer expands unceasingly, at the same time have already begun to will achieve 8051 and ARM kernel controller and a wireless transceiver, including ZIGBEE, Bluetooth, Wi-Fi transceiver integrated, such as with new forms of wireless MCU, get more and more widely used [1].

In today's era of the Internet of things, in colleges and universities and vocational schools, which is mainly composed of 8051 series MCU to reform the traditional teaching system with the new and urgent requirements, in colleges and universities and vocational schools for many years of accumulation of traditional MCU teaching on the teaching contents, teaching methods and the technical development of the disconnect, lack of timeliness, on the one hand, with the Internet of things technology integration, on the other hand also cannot effectively IoT professional curriculum system [1].

Based on extended sensor networks, wireless MCU and embedded technology and related rf microwave technology and high frequency technology as the foundation, discuss how to use the existing teaching system microprocessor, phase out old laboratory equipment, instrument by installing the latest teaching equipment, the technical point and the hardware platform, wireless MCU based on the latest spectral instrument and protocol analysis instrument, MCU teaching on the content, method, improvement and new train of thought of keeping pace with The Times.

At the same time put forward in the original teaching system integration for wireless MCU, embedded wireless technology, low-power technology, radio frequency circuits, antenna technology, aimed at improving SCM the efficiency of the teaching content and the Internet of things era of single-chip microcomputer teaching and practice teaching reform, improve the students' knowledge structure, cultivate outstanding engineers of the Internet of things era.

\section{The present status of the single-chip computer teaching}

Home is not only IoT professional equipped with single-chip computer classes, but almost all of the electronics and information engineering colleges vocational schools have opened the course microcontroller involved in electronics, communication, electrical, automation, electrical and other professional. For decades, the content of the single chip computer teaching are no big change, still with the single chip processor structure, instructions, assembly and so on, with a few simple microcontroller applications [2]. The SCM structure and instructions as the main line, do not accord with cognitive law should be subject, also does not conform to the current system on chip technology 
development trend, at present the mature and traditional teaching body is constant for a long time, how many technical problems have been unable to provide for the students to analyze, and already from two aspects of teaching ideas and technology development lag behind, must change.

From the teaching methods and experimental means, the current SCM old teaching methods, adopting traditional force-feeding teaching methods, dampened the enthusiasm of students to learn too much theory class, especially in the practice teaching seriously insufficient, lack of a large number of visualizations experiment means, and just use the old 8051 single chip microcomputer experiment box [2]. Can only play the role of demonstration, to cultivate students' ability to solve problems, brain, and now the Internet of things technology emphasizes the various means of communication, especially in wireless communications, the old SCM experiment equipment cannot satisfy completely the requirements of this new technology, at the same time, the less the experimental class and the course of theory and practice, and the innovation ability of students lack in single-chip microcomputer.

\section{The necessity of single chip microcomputer teaching reform}

IoT era to the single-chip microcomputer or embedded teaching reform provides opportunities and challenges. Internet of things era of single-chip microcomputer teaching in the teaching contents, teaching methods and curriculum system are put forward on the set of new urgent requirements [2]. For colleges and universities and vocational schools have courses in SCM, how in MCU course, expressed in networking related technologies? How IoT professional single-chip computer foundation course, and the Internet of things and other professional courses interface? How to adopt new teaching equipment and teaching means, improve the quality of teaching? How to use the Internet and related technology, single-chip computer teaching reform provides a rich teaching resources, contents and implementation methods? Answers to these questions itself also illustrates the single-chip microcomputer, the necessity of curriculum reform.

From the aspects of necessity, as can be seen from the single chip microcomputer teaching status quo of our country, no matter from the content to the current SCM teaching method needs to be changed. A reflection of students, learning attitude and learning effect also led to some extent it is necessary to change as soon as possible [1]. IoT development national strategy also requires universities to develop as soon as possible with the Internet of things technology, concept and application of design talent. Therefore, must be in basic course teaching in colleges and vocational schools in advance study of SOC single chip microcomputer and Internet connection, firmly grasp the basic knowledge and technology.

Feasible from the curriculum, on the Internet of things era involve embedded MCU teaching, modern communications, digital circuit, the network foundation, microcomputer principle and interface of single-chip computer, sensor and gathering, operating system and other basic courses and software and hardware knowledge and its related courses, divided into basic course, core curriculum and teaching practice, the application of development, many courses are already open courses. This is the basis of the single-chip computer teaching reform, reform, so, don't need to do to the existing curriculum system adjustment [3]. Core course, SOC single chip microcomputer can choose MSC 51, for the wireless MCU, also can choose more advanced ARM Cortex TM - A8 / M3 / M4 advanced 32 for SOC wireless MCU, it can be postpone, strengthening the traditional single-chip computer teaching. Embedded microcontroller processor and RTOS operating system is one of the courses in colleges and universities, here only need to use single chip microcomputer network gateway crop, can also be used as a IoT of routers, node, control center, network terminal. The teaching emphasizes the different role in the system of Internet of things. After the reform, MCU course can be opened as an independent course, also can be used as a IoT professional foundation courses. 


\section{Analysis of single-chip microcomputer teaching reform}

\subsection{The reform of curriculum content system structure.}

MCU teaching content has higher abstractness, this is the historical lineage. Therefore the course requirements for students speculative ability and flexibility of thinking is relative taller; Our current knowledge of relevant material in the relatively old, teaching content rarely embody the practical application of single-chip computer knowledge in related field, make learning to disconnect - both failed to reflect the modern education concept of "quality education", also did not reflect that the single chip microcomputer for each major "professional basic". In addition, in the actual teaching, the different students' different learning needs in MCU course has not received sufficient attention [3].

After the reform of course content, topic and task system structure of module, the entire course divided into modules, each module is composed of multiple subjects, each subject is composed of one or more tasks, each task by task goal, task analysis, relevant knowledge and task implementing four mutually coherent parts.

\subsection{Reform the teaching content organization.}

The influence of the traditional education idea, the current single-chip microcomputer classroom teaching methods and means of obvious lag behind. Especially in higher professional education under the new situation, how to develop the research of SCM learning method, provide targeted learning guide for students, stimulate students' interest in learning, cultivate the students' self-study ability, and should be to strengthen the teaching reform for discussion of one of the key content.

Constructivism believes that learning is the process of the students in their head to construct the meaning of the knowledge of the process. In the construction process, the student to the knowledge of cognitive cannot depend on the knowledge, is not on the books of the content of memory and to teacher thinking, but students complete the practice of the specific task due to the external environmental pressure in the process of its understanding and solve the problem, the resulting cognitive needs and learning motivation [4]. That is meaning construction is in some learning situation, by the student's own needs and the in the mind will gradually information processing or transformation and restructuring. Therefore, the teacher in the process of organizing teaching should follow the rules of the knowledge construction, to create a learning environment for students.

According to the theory of constructivism, reforming teaching content organization way, all around a mission objective of each teaching organization of each teaching link to the task as the main line, to the interpretation of the relevant knowledge and skill points. Task what knowledge and skills used in the points and then explain what knowledge and skills, and speak as much as they used, where use it where. Dominated by teachers to students as the center, talking and learn, learn and practice. Teachers' interpretation of the process, students are required to verify with related software on the computer, and ask some students on the platform for analysis and operation [4]. Real-time answers to questions, the students' classroom and homework questions, ask students to classroom teaching task circuit and program for local modification, to cultivate students SCM application circuit and the design method of the program.

\subsection{The reform of practical teaching design.}

Student experiment class teaching tasks as the object, with Protel software to map the circuit principle diagram, draw with Proteus software simulation circuit diagram, use WAVE input program and compile software or Keil software, then the program and the circuit simulation debugging operation [4]. The emphases and difficulties in the process of teachers on students' experiment, key points, and the problems of students in the process is operation in real time. And asked the students in the experimental circuit and program, on the basis of proper modification, cultivate their ability of independent design of circuit and program. Spare time ask students to experiment task, printed circuit board design and production, and the task program downloaded to the microcontroller, implement the task function. 


\subsection{Reform of the examination form.}

Course before examination form is relatively single, examination questions focused on memory, such as back a few instructions, said several parts of function, make a simple program, some students MCU test scores are high, but not the actual application. Examination form, therefore, the reform course of recovery course of vigor and role, and to adapt to the needs of the development of society, this is before us an important task of teaching reform.

\section{The Internet of things in the single chip computer teaching reform ideas and measures}

Because of the Internet of things is one of the core technology of micro power consumption under the condition of wireless communication technology, so the MCU and wireless communication together is the necessity of Internet of things era MCU teaching forms and characteristics of all kinds of new communications/protocol interface and the use of wireless single chip module is also a MCU or embedded system in the new period of the main form of expression [5]. For this, we need to combine the Internet of things technology development trend and the development of new teaching instrument and equipment, to the current teaching content, methods and practice links to change.

Teaching target hardware selected as more affordable wireless single chip (SOC). With the wireless MCU technology development, the wireless MCU kernel has 8051 so far, MSP430, the ARM architecture TM - M3 from 8 bits to the 32-bit kernel, such as single chip microcomputer can teaching from TI CC2530 this got started at present relatively mature ZIGBEE wireless MCU.

Teaching should be in the Internet of things applications and sensor network communications technology as the background, system network, wireless communication and data acquisition, radio frequency and antenna technology based micro power technology based new concept associated with the Internet of things [5]. Retain part teaching contents of traditional single chip microcomputer, such as $\mathrm{A} / \mathrm{D}, \mathrm{D} / \mathrm{A}$, serial communication, timer, SPI, I2C, interrupt technology and so on.

Software systems and network protocols, it is recommended that the choice of ZigBee and RF4CE protocol stack. Using the software instance and air protocol analyzer observe teaching the method to realize the layers in the protocol stack. Don't have to explain basic teaching stage RTOS operating system [6]. Software system is combined with network protocol part teaching only need simple interpretation of the test observation, should pay attention to training students' ability to practical use. Micro power technology is an important new technology of the sensor network, the traditional teaching of single-chip microcomputer and without this part of the content, however, use of router and nodes in sensor network, must work under the condition of micro power consumption, single chip computer teaching will increase the teaching content of this part of the technology.

Since at the same time, in order to realize wireless communication, it is impossible to leave the antennas and propagation, rf circuit, electromagnetic compatibility and so on some knowledge, it is impossible to leave frequency instrument use and measuring method of knowledge, this part of the original belongs to the complicated knowledge of radio frequency engineers, for the rf engineer must also be part of the knowledge, also need to add this part of knowledge to reform after the MCU teaching content.

In teaching methods, traditional single-chip computer parts, the adoption guide method with cases, and to the task as the center, creating environment, put forward to determine the trilogy of tasks, to complete the task to implement the teaching tasks; Corresponding to the new course, can improve for the sensor network application products as the center of the new train of thought, request application requirements, determine product design task, complete application products design test trilogy [6].

Teaching for wireless communication, the part in contact with the theory part is more complex, thus reducing the theory description and inference and increase the actual development and utilization, through the $9 \mathrm{k}$ - $6 \mathrm{GHZ}$ spectrum analyzer/signal generator and other advanced teaching equipment, let the student see the high frequency signal and high frequency power signal, learn how to use these instruments to carry out the survey and design of single chip microcomputer related circuit, communication distance and the wireless MCU module, signal quality, environmental noise and intuitive testing, enhance the students' perceptual knowledge, improve the students' interest in 
learning, lower part of the learning threshold for wireless communication [6].

For the quality of the network protocol recommendations to ZigBee and RF4CE blueprint, such as Keil development system using $C$ language to write the ZigBee protocol stack program for specific object, emphasize the process of application layer development, supplemented by the network layer and MAC layer of network in the form of different topologies in specific Settings. Since the realization of the simple point-to-point communication, gradually transition to the star network and network building. Gateway part can use common nodes and the communication; realize the Internet of things and Internet connectivity [5]. This section teaching method using the advanced graphical air protocol analyzer, can let students see real-time air flight of ZIGBEE protocol packaging, see the graphical network topology, packaging comparison, analysis, visualization and visual understanding of the connotation of protocol stack, greatly reduces the protocol stack teaching visual threshold and increase the teaching.

\section{Summary}

Teaching reform based on the single chip microcomputer as the breakthrough point and target, according to the "improve quality, do a characteristic" of the ministry of education requirements, select wireless microcontroller hardware platform and the advanced frequency instrument, protocol analysis and micro power consumption analysis instrument peer advanced experimental method, comprehensive reform of the traditional single-chip computer teaching. Internet of things or sensor network is one of the hot spot of the current university professional construction, under the background of Internet era, the reform of single-chip computer course teaching related hardware and software is feasible and necessary, the curriculum reform will help the reform of higher education and vocational education and advancing with The Times, help develop IoT industry needs the excellent engineer.

\section{References}

[1] J.X.Tian, An overview of the Internet of things, Nanjing: Southeast University, 2010 vol. 2, pp. 19-22.

[2] A.P. Bao, Single-chip computer application technology, Xi 'an: Xi 'an university of electronic science and technology journal, 2013, vol. 4, pp. 11-13.

[3] S.B. Shen, Internet architecture and related technology research, Journal of Nanjing University of posts and telecommunications, 2009, vol.6, pp.38-45.

[4] B.T. Li, MCS-51 single chip microcomputer application technology project tutorial, Beijing: Beijing University of aeronautics and astronautics press, 2011, vol.4, pp. 12-14.

[5] X.H. Cheng, Single-chip computer teaching reform, Low voltage apparatus, 2007, vol.4, pp.30-32.

[6] Sh. T. Miao, Internet management system research and design, Xi 'an: Xi 'an university of science and technology building, 2006, vol. 5, pp. 36-38. 